\title{
Probing the applicability of autotransporter based surface display with the EstA autotransporter of Pseudomonas stutzeri A15
}

Toon Nicolay, Lynn Lemoine, Elke Lievens, Sam Balzarini, Jos Vanderleyden and Stijn Spaepen*

\begin{abstract}
Background: Autotransporters represent a widespread family of secreted proteins in Gram-negative bacteria. Their seemingly easy secretion mechanism and modular structure make them interesting candidates for cell surface display of heterologous proteins. The most widely applied host organism for this purpose is Escherichia coli. Pseudomonas stutzeri A15 is an interesting candidate host for environmentally relevant biotechnological applications. With the recently characterized P. stutzeri A15 EstA autotransporter at hand, all tools for developing a surface display system for environmental use are available. More general, this system could serve as a case-study to test the broad applicability of autotransporter based surface display.

Results: Based on the P. stutzeri A15 EstA autotransporter $\beta$-domain, a surface display expression module was constructed for use in P. stutzeri A15. Proof of concept of this module was presented by successful surface display of the original EstA passenger domain, which retained its full esterase activity. Almost all of the tested heterologous passenger domains however were not exposed at the cell surface of P. stutzeri A15, as assessed by whole cell proteinase $\mathrm{K}$ treatment. Only for a beta-lactamase protein, cell surface display in P. stutzeri A15 was comparable to presentation of the original EstA passenger domain. Development of expression modules based on the full-length EstA autotransporter did not resolve these problems.

Conclusions: Since only one of the tested heterologous passenger proteins could be displayed at the cell surface of $P$. stutzeri A15 to a notable extent, our results indicate that the EstA autotransporter cannot be regarded as a broad spectrum cell surface display system in P. stutzeri A15.
\end{abstract}

Keywords: Autodisplay, Proteinase K, Secretion, Type V secretion system, Outer membrane protein, Beta barrel

\section{Background}

To cross the subsequent hurdles posed by the inner membrane, the periplasm and the outer membrane, Gram-negative bacteria have evolved at least seven different protein secretion mechanisms [1,2]. One widespread family of secreted proteins is constituted by autotransporters (ATs). These ATs represent a subset of the type $\mathrm{V}$ secretion system. This system is often regarded as the most simple secretion apparatus of Gram-negative bacteria. ATs possess a modular structure consisting of an $\mathrm{N}$-terminal signal peptide for targeting to the Sec translocon and subsequent translocation

\footnotetext{
* Correspondence: stijn.spaepen@biw.kuleuven.be

Centre of Microbial and Plant Genetics, KU Leuven, Kasteelpark Arenberg 20, 3001, Heverlee, Belgium
}

\section{Biomed Central

across the inner membrane, a passenger domain which represents the (often secreted) extracellular part and a C-terminal $\beta$-barrel domain which anchors the protein in the outer membrane. The passenger domain and the $\beta$-barrel domain are separated by an $\alpha$-helical linker domain [3]. The term AT was based on the initial view that no accessory proteins are required for transport of the passenger domain to the cell surface (and subsequent secretion). Yet, evidence shows that at least the Bam complex also fulfills an important role in this process [4].

Research in ATs originates from the role of most characterized ATs as virulence factors [5]. Apart from this, ATs are mainly of interest because replacement of the passenger domain with a heterologous protein makes them suitable candidates for cell surface display, a process termed autodisplay. Amongst others, it has been 
applied in vaccine development, library screening and whole-cell biocatalysis [6]. The most recent use is the extracellular accumulation of recombinant proteins $[7,8]$. Often this system is regarded as a universal and broadly applicable cell surface display system posing minimal requirements to the passenger domain $[6,8]$.

The biotechnological use of AT proteins has been mainly focused on Escherichia coli as host organism. For environmentally relevant biotechnological applications however, a more robust host is needed. The use of a different host organism for such applications is nevertheless limited to a few reports addressing metal remediation with Cupriavidus metallidurans $\mathrm{CH} 34$ [9,10] or Pseudomonas putida [11] for which the researchers applied the heterologous AT IgA of Neisseria gonorrhoeae. The use of an autologous AT, instead of a heterologous AT, might however be more appropriate. At least for $E$. coli, it has been suggested that the actual breakthrough for autodisplay has been initiated by the use of a for $E$. coli autologous AT [12].

Because of their involvement in environment-related processes, $P$. stutzeri strains represent good candidates for use in environmental applications [13]. We recently characterized the $P$. stutzeri A15 EstA AT [14], a close homologue of EstA of Pseudomonas aeruginosa PAO1 for which the full-length crystal structure is known [15]. They both are members of the GDSL ATs which represent a distinct family among the AT family. Features that distinguish their passenger domains from the majority of the ATs are their lipolytic activity, the permanent covalent attachment to the $\beta$-domain after surface display and the absence of the right-handed parallel $\beta$-helix structure [16]. An expression module based on the Pseudomonas putida EstA $\beta$ barrel domain was used to display a $\beta$-lactamase [17] and Pseudomonas and Burkholderia lipases at the cell surface of $E$. coli [18]. Becker et al. developed an expression module based on the entire EstA AT of $P$. aeruginosa for the cell surface display of lipolytic enzymes [19]. With the same expression module also a foldase was displayed at the cell surface [20].

We aimed to investigate the broad applicability of cell surface display of heterologous passenger domains using the AT system. As an interesting case-study we developed a P. stutzeri A15 EstA AT based expression module for the surface display of environmentally relevant proteins in $P$. stutzeri A15. This bacterium was isolated from the roots of paddy rice and later on classified as $P$. stutzeri $[21,22]$. The design of our $\beta$-domain based expression module could successfully be tested by the surface display of the original EstA passenger domain. This proof of concept contrasted with the encountered difficulties when heterologous passenger domains were used. We could show that the majority of these proteins were not displayed at the cell surface of $P$. stutzeri A15. The construction of two different expression modules based on the full-length EstA AT did not substantially solve these problems. Consequently, we conclude that the EstA AT cannot be regarded as a broad applicable cell surface display system in P. stutzeri A15.

\section{Results}

An EstA $\beta$-domain based expression module and the original EstA passenger domain as proof of concept

The recently published crystal structure of the EstA homologue of $P$. aeruginosa [15] served as a template to construct a structural model using the I-TASSER server [23] depicted in Figure 1A. The model allowed for the structurally informed design of two P. stutzeri A15 EstA based expression modules. Although still under debate $[24,25]$, recent research suggests that only the $\beta$-barrel and the $\alpha$-helical linker are sufficient for AT based surface display $[8,26]$. Therefore the designed expression modules, integrated in the pHERD26T backbone, contain a transport unit consisting of the $\beta$-barrel and the $\alpha$-helical linker (from residue A311 to L636, residue numbering starting at the GTG start codon; Est $\mathrm{A}_{\beta}$ on Figure 1B) of $P$. stutzeri A15 EstA as derived from the structural model. These expression modules also contain the P. stutzeri A15 EstA signal peptide. Furthermore two Sfil sites which allow directional cloning of different passenger domains and an E-epitope tag for detection were cloned between the signal peptide and the transport unit (Figure 1B). The signal peptide cleavage site was not affected compared to the wild type EstA when assessed by SignalP [27]. Both the SfiI sites and the E-epitope tag have previously been used to construct a full length $P$. aeruginosa system for the surface display of lipases [19]. The two expression modules were named $\mathrm{pEstA}_{\beta}$ and pEst $A_{\beta} L$, and express identical ORFs but differ at the $5^{\prime}$ untranslated region (UTR) preceding the ORF. The $5^{\prime}$ UTR of pEst $_{\beta} \mathrm{L}$ is identical to the $5^{\prime}$ UTR of pHERD26T-estA, which expresses the EstA AT, and was therefore expected to have similar expression levels as the latter. As a preliminary test, the P. stutzeri A15 EstAP passenger domain (from residue P27 to S325, EstAP on Figure 1B) was cloned as the passenger domain in the expression modules yielding pEst $_{\beta}$-est $A P$ and $\mathrm{pEstA}_{\beta} \mathrm{L}$-estAP. The EstAP fusion protein expressed from these plasmids only differs from EstA, which was expressed from pHERD26T-est $A$, by the presence of the E-epitope tag and the SfiI sites. A schematic representation of EstA derived from pHERD26T-estA and a fusion protein expressed from the expression modules pEst $_{\beta}$ and $p E s t A_{\beta} L$ is depicted in Figure $1 B$.

The EstAP fusion proteins derived from $\mathrm{pEst}_{\beta}$-estAP and pEst $_{\beta} \mathrm{L}$-estAP as well as EstA from pHERD26TestA were produced in $P$. stutzeri A15 cells. Both expression and cell surface display could be confirmed for all 


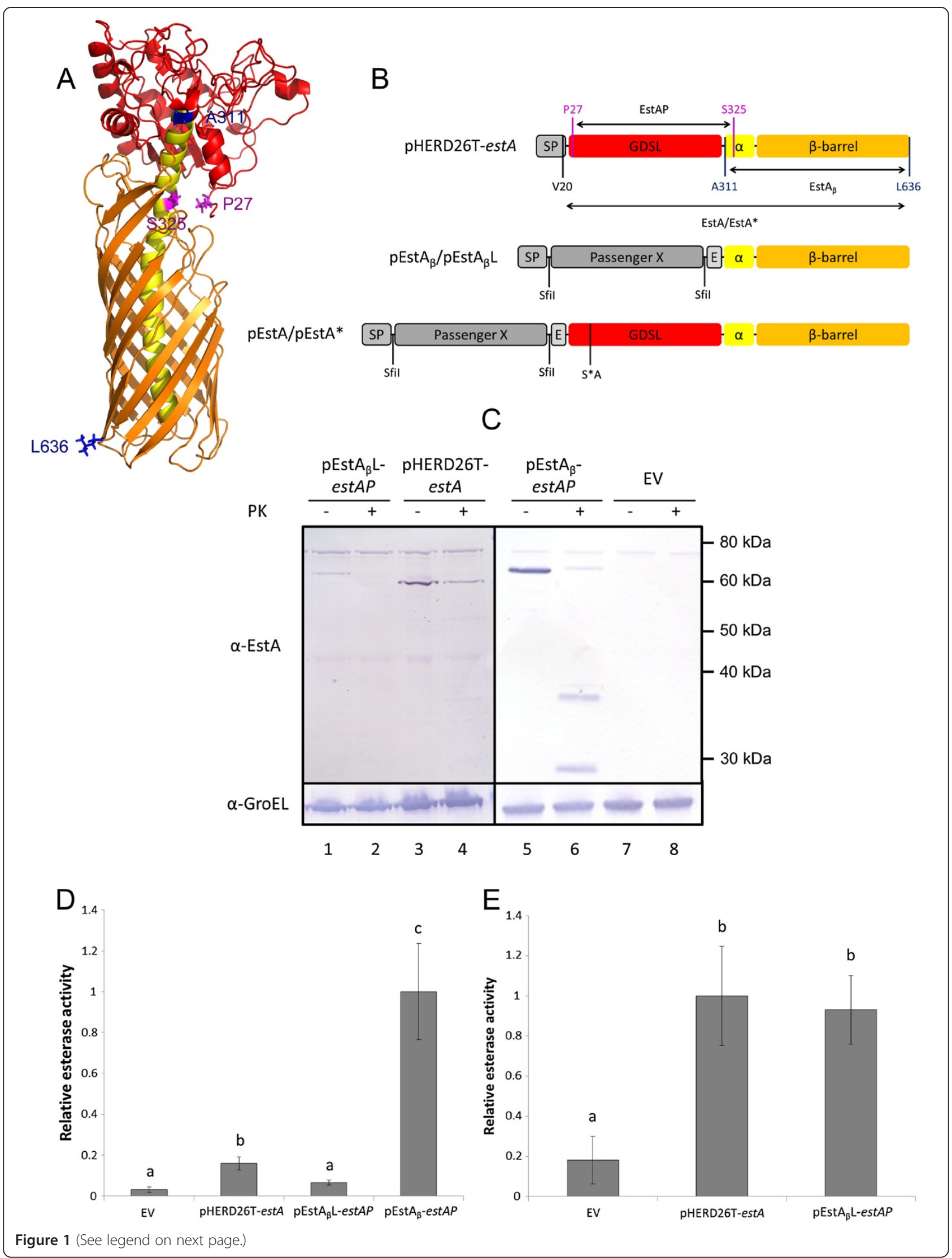


(See figure on previous page.)

Figure 1 Surface display of the EstA passenger domain. (A) Structural model of P. stutzeri A15 EstA (from D24 to L636) constructed using ITASSER [23]. (B) Schematic representation of proteins expressed from pHERD26T-estA (EstA), pEstA $A_{3} / p E s t A_{\beta} L$ and pEstA/pEstA*. (A-B) Residues delineating the part of EstA used for constructing pEst $A_{\beta} / p E s t A_{\beta} L\left(E s t A_{\beta}\right)$ are in blue ( $A 311$ and $\left.L 636\right)$. Residues marking the esterase passenger domain (EstAP) are coloured in magenta (P27 and S325). Residues for generating the full-length AT based constructs pEstA/pEstA* (V20, black and $L 636$, blue; see further) have been depicted. The difference between $p E s t A$ and $p E s t A^{*}$, mutagenesis of the catalytic serine residue ( $\left.S^{*} A\right)$, has been indicated. The GDSL esterase passenger domain (GDSL), the a-helical linker domain (a) and the $\beta$-barrel domain have been depicted in red, yellow and orange respectively, signal peptide (SP), E-epitope tag (E), Sfil restriction site (Sfil). (C) Induced cells of P. stutzeri A15 pHERD26T-estA, pEst $A_{\beta}$-estAP, $p E s t A_{\beta} L$-estAP or empty vector (EV) were treated with proteinase $\mathrm{K}(\mathrm{PK}+)$ or mock-treated $(\mathrm{PK}-)$ and analyzed with Western blot using anti-EstA serum (a-EstA) or anti-GroEL (a-GroEL). (D-E) Relative esterase activity of (D) cells of $P$. stutzeri A15 pHERD26T-estA, pEstAß-estAP, pEstA $A$ L- estAP or empty vector (EV) or (E) membrane fractions containing equal amounts of EstA/EstAP (Additional file 1: Figure S1). Data represent the mean of three independent repeats $\pm 95 \%$ confidence interval. The significance level $(P<0.05)$ as determined by one-way ANOVA with Student-Newman-Keuls post-hoc analysis, is indicated with a letter code. Molecular weight markers (kDa) are indicated at the side.

proteins by analyzing samples derived from whole cell proteinase $\mathrm{K}$ assays with SDS-PAGE and Western blot using $\alpha$-EstA serum (Figure 1C). Cell integrity in these samples was monitored by testing GroEL as a negative control protein. Furthermore, the functionality of the fusion proteins was tested by assessing the esterase activity of whole cells (Figure 1D). The data revealed the expected lower esterase activity of cells containing pHERD26T-estA or pEst $\mathrm{A}_{\beta} \mathrm{L}$-estAP as compared to cells containing $\mathrm{pEstA}_{\beta}$-estAP. This was expected since the $5^{\prime}$ UTR of these constructs is different. More surprising was the observation that the whole cell esterase activity differed between cells containing pHERD26T-est $A$ and pEs$\mathrm{tA}_{\beta} \mathrm{L}$-estAP. The esterase activity level of the latter did not even significantly differ from the empty vector (EV) negative control. Western blot analysis however revealed that the protein level of EstAP derived from pEst $\mathrm{A}_{\beta} \mathrm{L}$ estAP is much lower than the level of EstA (Figure $1 \mathrm{C}$ ). Moreover, the analysis of the esterase activity of membrane fractions (MFs) containing equal amounts of EstA or EstAP (Additional file 1: Figure S1) revealed that both proteins showed similar esterase activities (Figure 1E) and that this activity was membrane-associated.

Despite the similarities between the EstAP fusion proteins and EstA, we could observe a difference in stability of the passenger domain. We have previously shown that also the passenger domain of EstA and not only the $\beta$ barrel domain shows a heat modifiability shift, indicative of its relatively high stability [14]. Heat modifiability analysis showed that this shift could not be seen for the passenger domain of the EstAP fusion proteins. This is indicative of a less stable passenger domain in the EstAP fusion proteins (Additional file 2: Figure S2). The presence of the E-tag and the SfiI sites are likely to be responsible for the lowered stability of the passenger domain of EstAP compared to the one of EstA. Presumably they are also accountable for the difference in protein levels between pHERD26T-estA and pEst $\mathrm{A}_{\beta} \mathrm{L}$ containing cells.

Altogether these results indicate that the insertion of additional elements for the construction of an EstA based expression module do not abolish the functional surface display of the autologous passenger domain in $P$. stutzeri A15. The low protein levels obtained with pEs$t_{\beta} L$ favor the use of $\mathrm{pEstA}_{\beta}$ in further experiments.

\section{Limitations for cell surface display of heterologous passenger proteins}

Next, we intended to verify the universality of the pEs$\mathrm{tA}_{\beta}$ expression module. Hereto, the coding sequences of several different passenger domains were cloned into the Sfil sites of $\mathrm{pEstA}_{\beta}$ (Additional file 3: Table S1). Because of a general interest in addressing quorum sensing dependent biofilm formation, we assessed the cell surface display of three different lactonases: a Bacillus AiiA homologue named $\mathrm{AiiA}_{\text {soil }}$ and the related lactonases of Agrobacterium tumefaciens AiiB and AttM. To verify the more general applicability of the expression module also other proteins were examined: the fluorescent proteins mCherry, eGFP and yEVenus and the periplasmic protein Bla which has beta-lactamase activity.

Fusion protein production and cell surface display in P. stutzeri A15 was examined using whole cell proteinase $\mathrm{K}$ assays and subsequent analysis of the samples with SDS-PAGE and Western blot using $\alpha$-E-tag antibodies or $\alpha$-EstA serum (Figure 2A, B and Additional file 4: Figure S3). In most cases two different bands could be identified with a small difference in apparent molecular weight. The band with the higher apparent molecular weight (indicated with a '*' in Figure 2), which represented the majority of the fusion proteins, was inaccessible for proteinase $\mathrm{K}$. The band with the lower apparent molecular weight (indicated with an 'o' in Figure 2), which is much less abundant than the band with the higher molecular weight, was accessible for proteinase $\mathrm{K}$. Membrane fractions were isolated to assess the heat modifiability of these different bands (Figure 2C and D). The results showed that the band with the higher apparent molecular weight was not heat modifiable. In some cases the lower band did show a heat modifiability, in other cases this band is too faint to clearly visualize a potential heat modifiability shift. In combination with 


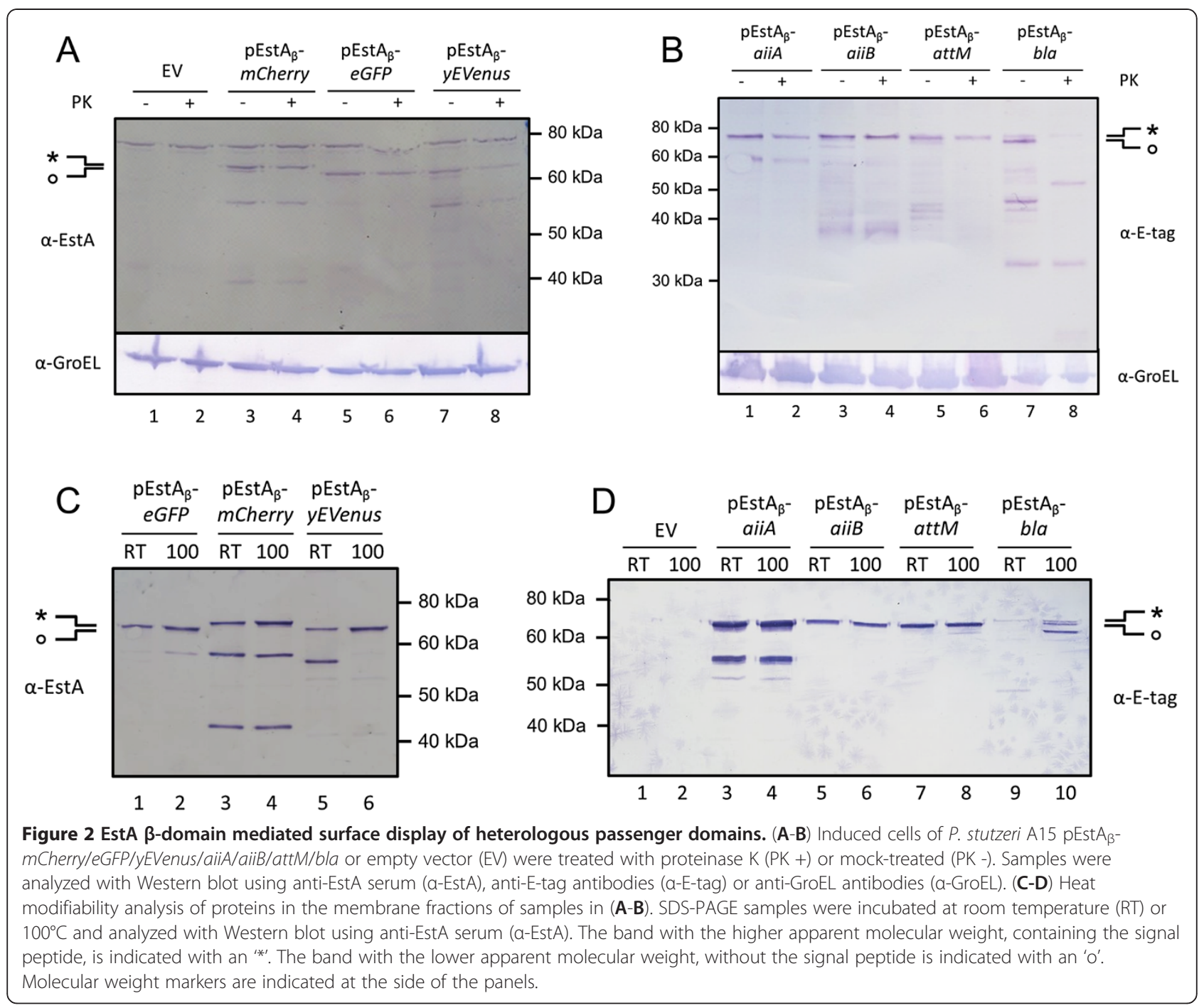

the evidence obtained from the whole cell proteinase $\mathrm{K}$ treatment assays this implies that the band with the lower apparent molecular weight was correctly folded in the MF and displayed at the cell surface. More importantly, the majority of the fusion proteins was not present in a correctly folded manner in the MF and was also not displayed at the cell surface. One notable exception was $\mathrm{pEstA}_{\beta}$-bla in which case the majority of the fusion protein was present as the band with the lower apparent molecular weight that was proteinase $\mathrm{K}$ accessible (Figure 2B, lanes 7 and 8) and heat modifiable (Figure 2D, lanes 9 and 10).

The most obvious explanation for the difference in molecular weight between the two protein bands is the presence of the signal peptide in the protein band with the highest molecular weight. Therefore, the presence of this protein band in the MF indicates that these proteins are stuck at the inner membrane or represent protein aggregates that are too small to be precipitated by the initial clearance step of the cell fractionation protocol.
Both explanations are in accordance with the absence of a heat modifiability shift of the proteins and their inaccessibility for proteinase K. In some cases also smaller sized specific protein bands could be identified, indicating either way translation intermediates or breakdown products.

Alltogether the results obtained for the heterologous passenger domains strongly differ from the results for the successfully displayed original EstA passenger domain. The results provide evidence that in almost all cases only a limited amount of the in P. stutzeri A15 expressed fusion proteins with a heterologous passenger domain can be displayed at the cell surface.

\section{Presence of the original AT passenger domain does not substantially improve surface display of heterologous passenger domains}

Because of the problems associated with the $\mathrm{pEstA}_{\beta}$ expression module we decided to construct a full-length 
AT based expression module. Moreover, recently published work suggested a promoting role for the autologous passenger domain in AT based surface display of heterologous passenger domains [25]. Also, previous work on surface display based on EstA of $P$. aeruginosa made use of a full-length AT based expression module $[19,20]$.

The pEstA and pEstA* expression modules consist of the full length EstA of $P$. stutzeri A15 (from residue V20 to residue L636, see Figure $1 \mathrm{~B}$ ). In the pEstA* plasmid, the catalytic serine of EstA was substituted for an alanine by site directed mutagenesis. Besides this mutation, both plasmids are identical to the $\mathrm{pEstA}_{\beta}$ plasmid containing the P. stutzeri A15 EstA signal peptide, two SfiI sites and an E-epitope tag. The same passenger domains as used for the pEst $_{\beta}$ experiments were cloned in pEstA and pEstA* (Additional file 3: Table S1). A schematic representation of a fusion protein expressed from these plasmids has been depicted in Figure 1B.

The production of the fusion proteins and their cell surface display in P. stutzeri A15 was assessed using whole cell proteinase $K$ assays and analysis of the samples with SDS-PAGE and Western blot (Figures 3A-B and Additional files 5: Figures S4-S7). In general, the results are similar to the ones obtained for the pEst $_{\beta}$ derived fusion proteins. Again, the bulk of the fusion proteins was inaccessible for proteinase K. When analyzing the MF, the inaccessible proteins also did not show a

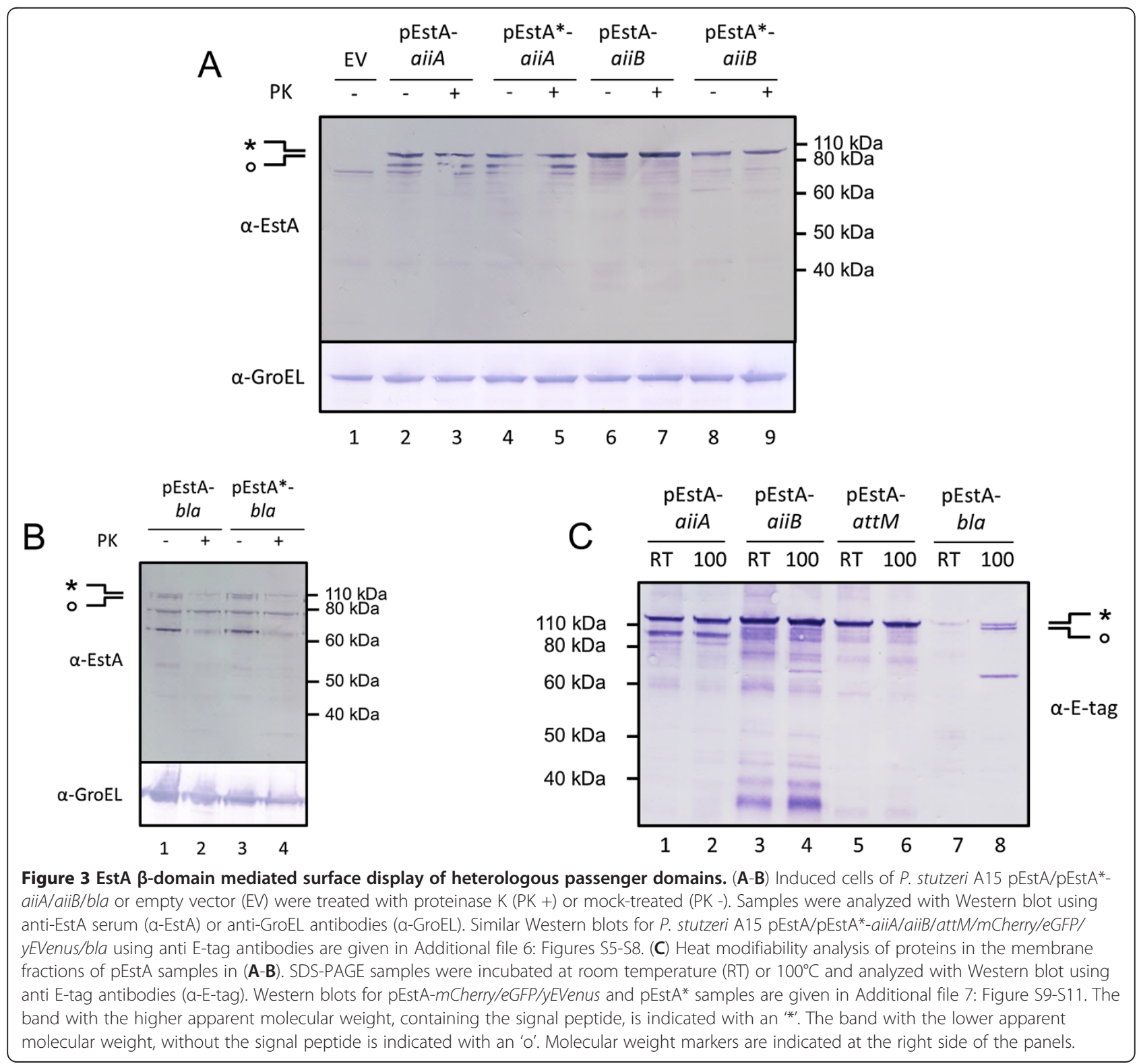


heat modifiability shift while the less abundant proteinase $\mathrm{K}$ accessible proteins did show a heat modifiability shift (Figures 3C and Additional files 6: Figures S8-S10). Also here, the exception was pEstA-bla/pEstA*-bla of which the greater part of the fusion proteins was properly folded in the MF (Figure 3C and Additional file 6: Figure S8) and displayed at the cell surface (Figure $3 \mathrm{~B}$ and Additional file 5: Figure S7). In general, lower bands that did not show heat modifiability presumably represent translation intermediates or breakdown products.

These data suggest that the problems associated with the EstA AT based surface display using the $\beta$-barrel domain in P. stutzeri A15 cannot substantially be alleviated by including the original passenger domain in the expression module.

\section{The beta-lactamase passenger domain is present in an enzymatically active conformation}

Surface display does not imply that the passenger domain is also in an active conformation. As described above (Figure 1D), the EstA passenger domain retained its esterase activity when displayed at the cell surface with the $\mathrm{pEstA}_{\beta}$ expression module. The whole cell proteinase $\mathrm{K}$ and heat modifiability assays showed that only the beta-lactamase passenger domain was substantially displayed at the cell surface of $P$. stutzeri A15. Therefore the beta-lactamase activity of whole cells containing the

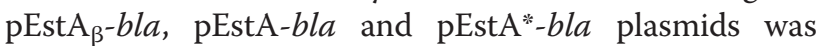
analyzed using nitrocefin as a substrate. This revealed that in all three cases a significant beta-lactamase activity could be detected as compared to the EV negative control and $\mathrm{pEstA}_{\beta}$-estAP (Figure 4).

\section{Discussion}

Up to now, various ATs have been used for the cell surface display of in total over thirty passenger domains [12]. In the past, the versatility and universality of AT based cell surface display has been emphasized $[6,28]$. Even more recently it was pointed out that this technology could be used for the extracellular accumulation of proteins with a diversity in size, structure and function [8]. With this in mind, we set out to test the broad applicability of AT based cell surface display using the recently characterized autologous EstA AT [14] in $P$. stutzeri A15 as a test-case.

In the first experiments, an expression module based on the EstA $\beta$-barrel and $\alpha$-helix was constructed. Whole cell proteinase $\mathrm{K}$ assays and esterase activity assays validated the expression module by showing the successful cell surface display of the original EstA passenger domain. Also cell surface display of a functional beta-lactamase as the passenger domain could be attained. So far, our results are in line with previous papers describing AT based cell surface display of esterases/lipases $[18,19,29,30]$ and a beta-lactamase [17] in E. coli. However, the same expression module failed to successfully display the majority of the fusion proteins with one of three different lactonases, Aii $_{\text {soil }}$, AiiB or AttM, as passenger domain. Also when one of the fluorescent proteins mCherry, eGFP or yEVenus was used as passenger domain, no successful cell surface display could be realized. Most reports focusing on passenger domain structure have looked at formation of disulfide bonds within the passenger domain in the periplasm and the compatibility with surface display. Recent research

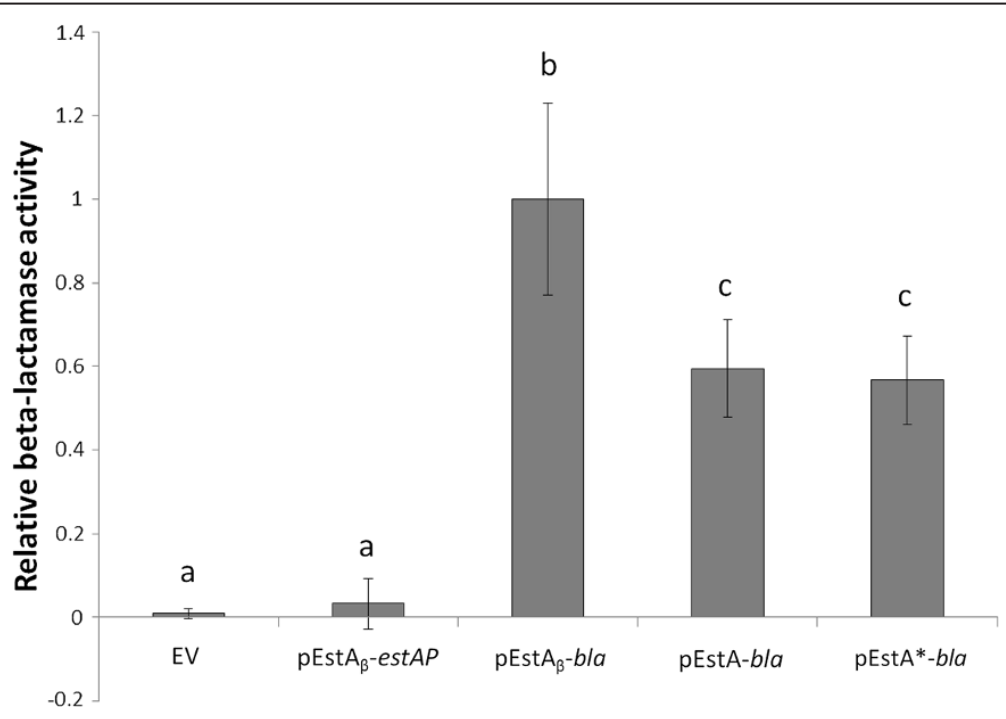

Figure 4 -lactamase activity of $P$. stutzeri A15 pEstA ${ }_{\beta}$-bla, pEstA-bla and pEstA ${ }^{*}$-bla. Relative $\beta$-lactamase activity of whole cells of $P$ stutzeri A15 pEstA $A_{\beta}$-estAP, pEstA $\beta_{\beta}$-bla, pEstA-bla, pEstA*-bla or empty vector (EV) assessed with nitrocefin as the substrate. Data represent the mean of three independent repeats $\pm 95 \%$ confidence interval. The significance level $(P<0.05)$ as determined by one-way ANOVA with StudentNewman-Keuls as post-hoc analysis is indicated with a letter code. 
seems to have reached a consensus on this point suggesting that only disulfide bonds between closely spaced cysteine residues in the passenger domain can be tolerated to achieve successful surface display [31,32]. The crystal structures of the fluorescent passenger domains reveal a $\beta$-barrel fold without disulfide bonds [33-35]. The same is true for the crystal structure of the AiiA lactonase of Bacillus thuringiensis [36] and on the basis of homology also for the lactonases used in this study. The only passenger domain that could successfully be surface displayed was a TEM $\beta$-lactamase. Interestingly, the crystal structure of the TEM-1 $\beta$ lactamase [37], contains one disulfide bond. The overall structure of this protein reveals a more globular structure which resembles most the structure of the original GDSL passenger domain. However, the globular structure is most likely not the main reason for surface display since $\beta$ lactamases have already been used as passenger domain for surface display using ATs with a completely different passenger domain structure $[25,38]$. Whereas the surface display of $\beta$-lactamases is in line with our findings, the display of the mCherry protein at the cell surface using the Pet AT [8] and the mRFP1 protein using the YfaL AT [7] is in contrast with our findings. In both studies $[7,8]$ cell surface display was achieved in $E$. coli.

A minimal translocation unit for AT based cell surface display, consisting of the $\beta$-barrel domain and the $\alpha$-helical linker domain, has been described for several ATs [39-43] including the EstA AT of P. putida [17]. More recently however, this original view was debated by the finding that the cell surface expression of heterologous proteins was increased when the full-length IcsA AT was used rather than only the $\beta$-domain [25]. This was supported by work on the Hbp AT where an intact $\beta$ stem was needed for efficient extracellular expression of the ESAT6 antigen [24].

Because of these reports and other publications in which a full-length EstA AT of $P$. aeruginosa was applied $[19,20]$ we designed full length EstA expression modules based on the EstA AT of P. stutzeri A15. Apart from the $\beta$-barrel domain and the $\alpha$-helical linker domain also the original esterase passenger domain was present in these modules, once with and once without the catalytic serine residue. The presence of the original passenger domain did not substantially resolve the problems encountered previously with the $\beta$-barrel domain based construct.

Also, in various cases smaller sized bands could be visualized. They could be indicative of translation intermediates or breakdown products. In E. coli it has been shown that surface exposed proteins can be proteolytically cleaved by the presence of proteases. Therefore, $E$. coli strains lacking the outer membrane protease (OmpT), like UT5600, are often preferred as host [12].

A first possible explanation for the differences between our results and reports describing successful surface display is the use of a different host organism. However, an often addressed issue for autodisplay in E. coli, the use of an for the host organism autologous AT, is nonetheless fulfilled [12]. Another reason could be the difference in viewpoint. Instead of focusing on the small fraction of passenger proteins that are proteinase $\mathrm{K}$ accessible and heat modifiable we concentrated on the major fraction of the proteins that were not secreted. The benchmark for successful surface display in our case was set by the data available for the surface display of the original EstA passenger domain using the $\beta$-barrel domain based construct. Others might have applied the term successful surface display in a less stringent way. Also, literature presumably has a bias towards the successful use of ATs for surface display. Despite the fact that the number of reports criticizing AT based surface display are limited, publications have reported the negative effect on membrane permeability [44] and on cell survival [45].

To end, it is important to mention that some reports are available which have addressed the nature of the passenger domain. The mechanism of translocation and secretion of passenger domains in the majority of these studies was only addressed by looking to the effects of introducing cysteine residues and the resulting disulfide bond formation. Some of the initial studies revealed the incompatibility of periplasmic disulfide bond formation with translocation of passenger domains [46-48]. These early reports were later contested by work describing the successful translocation of folded domains containing disulfide bonds $[26,49,50]$. The most recent studies on this topic however concluded that disulfide bond formation can only be tolerated if the cysteine residues are closely spaced [31,32]. Using a different approach it was suggested that the folding of a MalE passenger domain fused to the IgA AT interfered with its translocation across the outer membrane [51]. Also the introduction of the rigid and bulky calmodulin in the backbone of the Hbp AT has been shown to negatively affect secretion [32]. Finally it was proposed that secretion efficiency is dependent on the folding properties of the passenger domain itself [52]. Based on our results and the discussion above we suggest that more basic molecular knowledge of naturally occuring autotransporters is needed before such systems can be used for heterologous expression.

\section{Conclusion}

We have shown that an expression module based on the $\beta$-domain of the $P$. stutzeri A15 EstA AT is not generally applicable for successful surface display of heterologous passenger domains in P. stutzeri A15. Essentially, the same holds true for expression modules based on the full-length EstA AT. In conclusion, the EstA AT cannot 
be seen as a broad applicable cell surface display system in P. stutzeri A15.

\section{Methods}

\section{Bacterial strains and growth conditions}

E. coli strain Top10 (Life Technologies) was used for cloning. For protein expression, P. stutzeri strain A15 $[21,22]$ was used. Triparental conjugation of plasmids to P. stutzeri A15 was carried out with the helper plasmid pRK2073.

P. stutzeri A15 and E. coli strains were routinely cultured as described before [14]. If appropriate, antibiotics were added to the media in a final concentration of 10 $\mu \mathrm{g} \mathrm{ml}^{-1}$ for tetracycline (Tc) and $50 \mu \mathrm{g} \mathrm{ml}^{-1}$ for spectinomycin (Sp).

\section{Construction of plasmids and general molecular biological techniques}

All restriction enzymes (New England Biolabs), T4 DNA ligase (Life Technologies), Pfx DNA polymerase (Life Technologies), GenElute HP Plasmid Miniprep Kit (Sigma-Aldrich), GenElute PCR Clean Up Kit (SigmaAldrich), QIAEX II Gel Extraction Kit (Qiagen) and Puregene Genomic DNA Isolation Kit (Qiagen) were used according to the manufacturer's instructions. Oligonucleotide primers were purchased from IDT DNA. All plasmids were sequenced by GATC Biotech to verify the nucleotide sequences.

All plasmids and primers used in this study are listed in Table S1 (Additional file 3) and Table S2 (Additional file 7), respectively. The plasmids constructed in this study have a pHERD26T [53] backbone. To construct the $\mathrm{pEstA}_{\beta}$ plasmid a number of intermediate constructs was created. The $\beta$-domain and part of the $\alpha$-helical linker (from residue W328 to residue L636, residue numbering starting at the GTG start codon) of estA were PCR amplified with primers AI2981 and AI2982. This fragment was cloned in the HindIII/XbaI sites of pHERD26T. In the next step, the nucleotide sequence coding for the EstA signal peptide, 2 Sfil sites and the Etag were introduced into the intermediary plasmid. Hereto a pUC19 fragment was PCR amplified using primers AI3505 and AI3507 and cloned into the vector in the XbaI/NcoI sites. The original pHERD26T SfiI site was furthermore removed by site directed mutagenesis with primers AI4569, AI4570, AI4571 and AI4572. Because this intermediary plasmid did not possess the entire $\alpha$-helical linker, a new estA fragment was PCR amplified with primers AI4985 and AI4986 and cloned in the $\mathrm{XbaI} / \mathrm{SalI}$ sites. This resulted in the $\mathrm{pEstA}_{\beta}$ plasmid (with the $\alpha$-helical linker starting from residue A311). To construct pEst $\mathrm{A}_{\beta} \mathrm{L}$, the 5' UTR of pHERD26T-estA was PCR amplified with primers AI7328 and AI7329 and cloned into the $\mathrm{XbaI} / \mathrm{NcoI}$ sites of $\mathrm{pEstA}_{\beta}$.
In the first step to create the pEstA and pEstA* plasmids an estA fragment without the signal peptide (from residue V20 to residue L636) was PCR amplified using primers AI2982 and AI2984. The PCR product was cloned in the HindIII/XbaI sites of pHERD26T. Hereafter the nucleotide sequence coding for the EstA signal peptide, 2 SfiI sites and the E-tag were added to the plasmid. This was done by PCR amplification of a pUC19 spacer fragment using the primers AI4184 and AI3507 and subsequent cloning in the $\mathrm{XbaI} / \mathrm{EcoRI}$ sites. Also here the pHERD26T original Sfil site was afterwards removed by site directed mutagenesis. Because of a different cloning strategy the $5^{\prime}$ UTR of the resulting plasmid differs from the one present in $\mathrm{pEstA}_{\beta}$. To get similar expression levels for both plasmid series the pEs$\mathrm{tA}_{\beta} 5^{\prime}$ UTR was also used for this plasmid series. In a first step a silent mutation removed the NcoI site present in the estA fragment of the plasmid using primers AI6276, AI6277, AI6278 and AI6280. In the resulting vector the $\mathrm{XbaI} / \mathrm{NcoI}$ fragment was replaced by the $\mathrm{XbaI} / \mathrm{NcoI}$ fragment of $\mathrm{pEst}_{\beta}$ resulting in $\mathrm{pEstA}$. In this plasmid the catalytic serine of EstA was substituted for an alanine by site directed mutagenesis using primers AI5083, AI5084, AI5085 and AI6280. This gave rise to pEstA*.

To create $\mathrm{pEstA}_{\beta}$-aiiA, $\mathrm{pEstA}_{\beta}$-aiiB, $\mathrm{pEstA}_{\beta}$-attM, $\mathrm{pEstA}_{\beta}-e G F P, \quad \mathrm{pEstA}_{\beta}-m$ Cherry, $\mathrm{pEstA}_{\beta}-y$ EVenus and pEst $A_{\beta}$-bla the coding sequences of respectively aiiA, aiiB, attM, eGFP, mCherry, yEVenus and bla were PCR amplified with the respective primer pairs AI4567/ AI4568, AI4958/AI4959, AI4956/AI4957, AI5247/ AI5248, AI5247/AI5248, AI5245/AI5246 and AI7193/ AI7194 on the respective DNA templates pMIR101, Agrobacterium tumefaciens C58 gDNA, Agrobacterium tumefaciens C58 gDNA, pET28a-eGFP, pET28a-mCherry, pKT103 and pUC19. The resulting fragments were cloned into the Sfil sites of $\mathrm{pEstA}_{\beta}$. In a similar way pEstA-aiiA, pEstA*-aiiA, pEstA-aiiB, pEstA*-aiiB, pEstA-attM, pEstA"-attM, pEstA-eGFP, pEstA*-eGFP, pEstA-mCherry, pEstA*-mCherry, pEstA-yEVenus, pEstA ${ }^{*}-y E V e n u s$, pEstA-bla and pEstA*-bla were constructed. $\mathrm{pEstA}_{\beta}$-estAP, $\mathrm{pEstA-estAP}$ and $\mathrm{pEstA}^{*}$-estAP were made by cloning the coding sequence of the esterase passenger domain of estA (from residue P27 to S325) into $\mathrm{pEstA}_{\beta}$, pEstA and pEstA* respectively.

\section{Protein expression and analysis}

Expression of ORFs on the pHERD26T derivatives was under control of the $\mathrm{P}_{\mathrm{BAD}}$ promoter. P. stutzeri A15 strains were grown at $30^{\circ} \mathrm{C}$ and $200 \mathrm{rpm}$ in $\mathrm{LB}+\mathrm{Tc}$ until $\mathrm{OD}_{600}$ of 0.8 was reached and $0.2 \% \mathrm{~L}$-arabinose (final concentration) was added to start protein production. After induction, growth was continued at $23^{\circ} \mathrm{C}$ and 200 rpm for 3 hours. 
For whole cell proteinase $\mathrm{K}$ digestion assays, $1.5 \mathrm{ml}$ samples were resuspended in buffer $(50 \mathrm{mM}$ Tris $\mathrm{pH} 8$, $7.5 \mathrm{mM} \mathrm{CaCl}_{2}$ ) to obtain an $\mathrm{OD}_{600}$ of $5.100 \mu \mathrm{l}$ samples were treated with a final concentration of $100 \mathrm{ng} \mathrm{Ll}^{-}$ ${ }^{1}$ proteinase $\mathrm{K}$ (Fischer) or mock treated with distilled water and incubated for one hour at $37^{\circ} \mathrm{C}$ and $1400 \mathrm{rpm}$. To stop digestion $4 \mathrm{mM}$ phenylmethanesulfonylfluoride was added. After centrifugation, the cell pellet was resuspended in $105 \mu \mathrm{l}$ buffer (50 mM Tris $\mathrm{pH} 8,4 \mathrm{mM}$ phenylmethanesulfonylfluoride).

To test for beta-lactamase activity, induced cells were resuspended in $0.85 \% \mathrm{NaCl}$ to obtain an $\mathrm{OD}_{600}$ of 1.5 . A $0.5 \mathrm{mg} \mathrm{ml}^{-1}$ nitrocefin stock solution $(2 \mathrm{mg}$ nitrocefin, Calbiochem) was suspended in $1.9 \mathrm{ml} 100 \mathrm{mM}$ MOPS, $0.85 \% \mathrm{NaCl}, \mathrm{pH} 7$ and $0.1 \mathrm{ml} \mathrm{DMSO}$ and was twentyfold diluted in $100 \mathrm{mM}$ MOPS, $0.85 \% \mathrm{NaCl}, \mathrm{pH} 7.90 \mu \mathrm{l}$ was added to $10 \mu \mathrm{l}$ of the bacterial suspension. The activity was measured by monitoring $\mathrm{OD}_{486}$ at $37^{\circ} \mathrm{C}$. Esterase activity of whole cells or MFs was measured as described before [14]. MFs were prepared starting from $200 \mathrm{mg}$ cell pellet as previously described [14].

For visualization, proteins were separated by SDSPAGE (NuPAGE 10\% Bis-Tris MOPS, Life Technologies) and detected with Western blotting. Prior to SDS-PAGE, samples were heated $10 \mathrm{~min}$ at $70^{\circ} \mathrm{C}$ unless otherwise stated. After electrophoresis, proteins were transferred onto polyvinylidene fluoride membranes (Life Technologies) by electroblotting $\left(30 \mathrm{~V} / 60 \mathrm{~min}\right.$ at $\left.4^{\circ} \mathrm{C}\right)$. Membranes were incubated with the previously described rabbit anti-EstA serum (1:2000 dilution) [14], rabbit anti E-tag antibodies (1:2000 dilution, Abcam) or with rabbit antiGroEL antibodies (1:2000 dilution, Sigma-Aldrich). Detection was done via alkaline-phosphatase conjugated goat anti-rabbit IgG secondary antibodies (SigmaAldrich) and NBT/BCIP (Nitro blue tetrazolium chloride/5-Bromo-4-chloro-3-indolylphosphate, Sigma).

\section{Additional files}

Additional file 1: Figure S1. Assessment of the fusion protein concentration in the membrane fractions of $P$. stutzeri A15 pHERD26TestA or pEstAßL-estAP.

Additional file 2: Figure S2. Heat modifiability analysis of proteins in the membrane fractions of $P$. stutzeri A15 pHERD26T-estA, pEstA $\beta_{\beta}$-estAP and $p$ Est $A_{\beta} L$-estAP.

Additional file 3: Table S1. Plasmids used in this study.

Additional file 4: Figure S3. Proteinase $K$ accessibility of fusion proteins

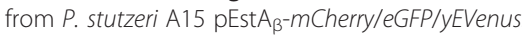

Additional file 5: Figures S4-S7. Proteinase $K$ accessibility of fusion proteins from P. stutzeri A15 pEstA/pEstA*-aiiA/aiiB/attM/eGFP/mCherry/ yEVenus/bla.

Additional file 6: Figures S8-S10. Heat modifiability analysis of proteins in the membrane fractions of $P$. stutzeri A15 pEstA*-aiiA/ aiiB/attM/bla/eGFP/mCherry/yEVenus and pEstA-eGFP/mCherry/ yEVenus

Additional file 7: Table S2. Primers used in this study.

\section{Abbreviations}

AT: Autotransporter; EstAP: EstA passenger domain; EV: Empty Vector MF: Membrane Fraction; PK: Proteinase K; UTR: Untranslated Region.

\section{Competing interests}

The authors declare that they have no competing interests.

\section{Authors' contributions}

T.N., L.L, E.L. and S.B. designed and constructed the expression plasmids. T.N performed enzymatic assays, membrane preparations and whole cell proteinase $\mathrm{K}$ assays. T.N., J.V. and S.S. designed the research project and wrote the manuscript. All authors read and approved the final manuscript.

\section{Acknowledgements}

The pHERD26T vector was a gift from H.D. Yu (Marshall University). T.N. and S.S. are recipients of a doctoral and post-doctoral fellowship grant respectively from Research Foundation Flanders (FWO-Vlaanderen).

Received: 26 October 2012 Accepted: 11 December 2012

Published: 13 December 2012

\section{References}

1. Economou A, Christie PJ, Fernandez RC, Palmer T, Plano GV, Pugsley AP: Secretion by numbers: Protein traffic in prokaryotes. Mol Microbiol 2006, 62(2):308-319.

2. Abdallah MA, Van Pittius NCG, Champion PAD, Cox J, Luirink J, Vandenbroucke-Grauls CMJE, Appelmelk BJ, Bitter W: Type VII secretion mycobacteria show the way. Nat Rev Microbiol 2007, 5(11):883-891.

3. Dautin N, Bernstein HD: Protein secretion in Gram-negative bacteria via the autotransporter pathway. Annu Rev Microbiol 2007, 61:89-112.

4. Leyton DL, Rossiter $A E$, Henderson IR: From self sufficiency to dependence: mechanisms and factors important for autotransporter biogenesis. Nat Rev Microbiol 2012, 10(3):213-225.

5. Nishimura K, Tajima N, Yoon Y-H, Park S-Y, Tame JRH: Autotransporter passenger proteins: virulence factors with common structural themes. $J$ Mol Med-Jmm 2010, 88(5):451-458.

6. Jose J, Meyer TF: The autodisplay story, from discovery to biotechnical and biomedical applications. Microbiol Mol Biol R 2007, 71(4):600-619.

7. Ko H-J, Park E, Song J, Yang TH, Lee HJ, Kim KH, Choi I-G: Functional cell surface display and controlled secretion of diverse agarolytic enzymes by Escherichia coli with a novel ligation-independent cloning vector based on the autotransporter YfaL. Appl Environ Microbio/ 2012, 78(9):3051-3058.

8. Sevastsyanovich YR, Leyton DL, Wells TJ, Wardius CA, Tveen-Jensen K, Morris FC, Knowles TJ, Cunningham AF, Cole JA, Henderson IR: A generalised module for the selective extracellular accumulation of recombinant proteins. Microb Cell Fact 2012, 11:69.

9. Biondo R, da Silva FA, Vicente EJ, Souza Sarkis JE, Schenberg AC: Synthetic phytochelatin surface display in Cupriavidus metallidurans $\mathrm{CH} 34$ for enhanced metals bioremediation. Environ Sci Technol 2012, 46(15):8325-8332.

10. Valls $M$, Atrian $S$, de Lorenzo V, Fernandez LA: Engineering a mouse metallothionein on the cell surface of Ralstonia eutropha $\mathrm{CH} 34$ for immobilization of heavy metals in soil. Nat Biotechnol 2000, 18(6):661-665.

11. Valls M, de Lorenzo V, Gonzalez-Duarte R, Atrian S: Engineering outermembrane proteins in Pseudomonas putida for enhanced heavy-metal bioadsorption. J Inorg Biochem 2000, 79(1-4):219-223.

12. Jose J, Maas RM, Teese MG: Autodisplay of enzymes-Molecular basis and perspectives. J Biotechnol 2012, 161(2):92-103.

13. Garcia-Valdes E, Mulet M, Lalucat J: Insights into the life styles of Pseudomonas stutzeri. In Pseudomonas, Volume 6. 1st edition. Edited by Ramos JL, Filloux A. New York: Springer; 2010:177-198.

14. Nicolay T, Devleeschouwer K, Vanderleyden J, Spaepen S: Characterization of Esterase A, a Pseudomonas stutzeri A15 autotransporter. Appl Environ Microbiol 2012, 78(8):2533-2542.

15. van den Berg B: Crystal structure of a full-length autotransporter. J Mol Biol 2011, 396(3):627-633.

16. Wilhelm S, Rosenau F, Kolmar H, Jaeger K-E: Autotransporters with GDSL passenger domains: Molecular physiology and biotechnological applications. ChemBioChem 2011, 12(10):1476-1485. 
17. Yang TH, Pan JG, Seo YS, Rhee JS: Use of Pseudomonas putida EstA as an anchoring motif for display of a periplasmic enzyme on the surface of Escherichia coli. Appl Environ Microbiol 2004, 70(12):6968-6976.

18. Yang TH, Kwon M-A, Song JK, Pan JG, Rhee JS: Functional display of Pseudomonas and Burkholderia lipases using a translocator domain of EstA autotransporter on the cell surface of Escherichia coli. J Biotechnol 2010, 146(3):126-129.

19. Becker S, Theile S, Heppeler N, Michalczyk A, Wentzel A, Wilhelm S, Jaeger $\mathrm{KE}$, Kolmar H: A generic system for the Escherichia coli cell-surface display of lipolytic enzymes. FEBS Lett 2005, 579(5):1177-1182.

20. Wilhelm S, Rosenau F, Becker S, Buest S, Hausmann S, Kolmar H, Jaeger KE: Functional cell-surface display of a lipase-specific chaperone. ChemBioChem 2007, 8(1):55-60.

21. Vermeiren $H$, Willems A, Schoofs $G$, de Mot R, Keijers $V$, Hai WL, Vanderleyden J: The rice inoculant strain Alcaligenes faecalis A15 is a nitrogen-fixing Pseudomonas stutzeri. Syst Appl Microbiol 1999, 22(2):215-224

22. You CB, Song W, Wang HX, Li JP, Lin M, Hai WL: Associative nitrogen fixation of Alcaligenes faecalis with rice plant. Biol Nitrogen Fix Newsl Sydney Univ 1983, 11:92-103.

23. Zhang Y: I-TASSER server for protein 3D structure prediction. BMC Bioinforma 2008, 9:40.

24. Jong WS, Soprova Z, de Punder K, Ten Hagen-Jongman CM, Wagner S, Wickstrom D, de Gier JW, Andersen P, van der Wel NN, Luirink J: A structurally informed autotransporter platform for efficient heterologous protein secretion and display. Microb Cell Fact 2012, 11(1):85.

25. Lum M, Morona R: IcsA autotransporter passenger promotes increased fusion protein expression on the cell surface. Microb Cell Fact 2012, 11:20.

26. Marin E, Bodelon G, Fernandez LA: Comparative analysis of the biochemical and functional properties of C-terminal domains of autotransporters. J Bacteriol 2010, 192(21):5588-5602.

27. Petersen TN, Brunak S, von Heijne G, Nielsen H: SignalP 4.0: discriminating signal peptides from transmembrane regions. Nat Meth 2011, 8(10):785-786

28. Kjaergaard K, Hasman H, Schembri MA, Klemm P: Antigen 43-mediated autotransporter display, a versatile bacterial cell surface presentation system. J Bacteriol 2002, 184(15):4197-4204.

29. Schultheiss E, Paar C, Schwab H, Jose J: Functional esterase surface display by the autotransporter pathway in Escherichia coli. J Mol Catal B: Enzym 2002, 18(1-3):89-97.

30. Schultheiss E, Weiss S, Winterer E, Maas R, Heinzle E, Jose J: Esterase autodisplay: Enzyme engineering and whole-cell activity determination in microplates with pH sensors. Appl Environ Microbiol 2008, 74(15):47824791.

31. Leyton DL, Sevastsyanovich YR, Browning DF, Rossiter AE, Wells TJ, Fitzpatrick RE, Overduin M, Cunningham AF, Henderson IR: Size and conformation limits to secretion of disulfide-bonded loops in autotransporter proteins. J Biol Chem 2011, 286(49):42283-42291.

32. Jong WSP, ten Hagen-Jongman CM, den Blaauwen T, Slotboom DJ, Tame JRH, Wickstrom D, de Gier J-W, Otto BR, Luirink J: Limited tolerance towards folded elements during secretion of the autotransporter $\mathrm{Hbp}$. Mol Microbiol 2007, 63(5):1524-1536.

33. Shu X, Shaner NC, Yarbrough CA, Tsien RY, Remington SJ: Nove chromophores and buried charges control color in mFruits. Biochemistry US 2006, 45(32):9639-9647.

34. Yang F, Moss LG, Phillips GN: The molecular structure of green fluorescent protein. Nat Biotechnol 1996, 14(10):1246-1251.

35. Rekas A, Alattia J-R, Nagai T, Miyawaki A, Ikura M: Crystal structure of venus, a yellow fluorescent protein with improved maturation and reduced environmental sensitivity. J Biol Chem 2002, 277(52):50573-50578.

36. Kim MH, Choi W-C, Kang HO, Lee JS, Kang BS, Kim K-J, Derewenda ZS, Oh $\mathrm{T}-\mathrm{K}$, Lee $\mathrm{CH}$, Lee J-K: The molecular structure and catalytic mechanism of a quorum-quenching $\mathrm{N}$-acyl-L-homoserine lactone hydrolase. P Natl Acad Sci USA 2005, 102(49):17606-17611.

37. Jelsch C, Mourey L, Masson JM, Samama JP: Crystal structure of Escherichia coli TEM1 beta-lactamase at 1.8 A resolution. Proteins 1993, 16(4):364-383.

38. Lattemann CT, Maurer J, Gerland E, Meyer TF: Autodisplay: Functional display of active beta-lactamase on the surface of Escherichia coli by the AIDA-I autotransporter. J Bacterio/ 2000, 182(13):3726-3733.

39. Klauser T, Kramer J, Otzelberger K, Pohlner J, Meyer TF: Characterization of the Neisseria $\lg \mathrm{A}$ (beta) core: The essential unit for outer-membrane targeting and extracellular protein secretion. J Mol Biol 1993, 234(3):579593.

40. Suzuki T, Lett MC, Sasakawa C: Extracellular transport of VirG protein in Shigella. J Biol Chem 1995, 270(52):30874-30880.

41. Maurer J, Jose J, Meyer TF: Characterization of the essential transport function of the AIDA-I autotransporter and evidence supporting structural predictions. J Bacterio/ 1999, 181(22):7014-7020.

42. Oliver DC, Huang G, Fernandez RC: Identification of secretion determinants of the Bordetella pertussis BrkA autotransporter. J Bacteriol 2003, 185(2):489-495.

43. St Geme JW, Cutter D: The Haemophilus influenzae Hia adhesin is an autotransporter protein that remains uncleaved at the $\mathrm{C}$ terminus and fully cell associated. J Bacteriol 2000, 182(21):6005-6013.

44. Van Gerven N, Sleutel M, Deboeck F, De Greve H, Hernalsteens JP: Surface display of the receptor-binding domain of the F17a-G fimbrial adhesin through the autotransporter AIDA-I leads to permeability of bacterial cells. Microbiol-Sgm 2009, 155:468-476.

45. Binder U, Matschiner G, Theobald I, Skerra A: High-throughput sorting of an anticalin library via EspP-mediated functional display on the Escherichia coli cell surface. J Mol Biol 2010, 400(4):783-802.

46. Jose J, Kramer J, Klauser T, Pohlner J, Meyer TF: Absence of periplasmic DsbA oxidoreductase facilitates export of cysteine-containing passenger proteins to the Escherichia coli cell surface via the $\operatorname{lgA}($ beta) autotransporter pathway. Gene 1996, 178(1-2):107-110.

47. Klauser T, Pohlner J, Meyer TF: Extracellular transport of Cholera-toxin Bsubunit using Neisseria IgA protease beta-domain: Conformationdependent outer-membrane translocation. EMBO J 1990, 9(6):1991-1999.

48. Klauser T, Pohlner J, Meyer TF: Selective extracellular release of Cholera toxin-B subunit by Escherichia coli: Dissection of Neisseria IgA-betamediated outer-membrane transport. EMBO J 1992, 11(6):2327-2335.

49. Veiga $E$, de Lorenzo V, Fernandez LA: Structural tolerance of bacterial autotransporters for folded passenger protein domains. Mol Microbiol 2004, 52(4):1069-1080.

50. Skillman KM, Barnard TJ, Peterson JH, Ghirlando R, Bernstein HD: Efficient secretion of a folded protein domain by a monomeric bacterial autotransporter. Mol Microbiol 2005, 58(4):945-958.

51. Rutherford N, Charbonneau ME, Berthiaume F, Betton JM, Mourez M: The periplasmic folding of a cysteineless autotransporter passenger domain interferes with its outer membrane translocation. J Bacterio/ 2006, 188 (11):4111-4116.

52. Renn JP, Junker M, Besingi RN, Braselmann E, Clark PL: ATP-independent control of autotransporter virulence protein transport via the folding properties of the secreted protein. Chem Biol 2012, 19(2):287-296.

53. Qiu DR, Damron FH, Mima T, Schweizer HP, Yu HD: P-BAD-based shuttle vectors for functional analysis of toxic and highly regulated genes in Pseudomonas and Burkholderia spp. and other bacteria. Appl Environ Microbiol 2008, 74(23):7422-7426.

\section{doi:10.1186/1475-2859-11-158}

Cite this article as: Nicolay et al:: Probing the applicability of autotransporter based surface display with the EstA autotransporter of Pseudomonas stutzeri A15. Microbial Cell Factories 2012 11:158.

\section{Submit your next manuscript to BioMed Central and take full advantage of:}

- Convenient online submission

- Thorough peer review

- No space constraints or color figure charges

- Immediate publication on acceptance

- Inclusion in PubMed, CAS, Scopus and Google Scholar

- Research which is freely available for redistribution 